Mathematical Modelling and Analysis

Volume 21 Number 4, July 2016, 478-501

http://dx.doi.org/10.3846/13926292.2016.1183527

(c) Vilnius Gediminas Technical University, 2016
Publisher: Taylor\&Francis and VGTU

http://www.tandfonline.com/TMMA

ISSN: $1392-6292$

eISSN: $1648-3510$

\title{
Parallel Extragradient-Proximal Methods for Split Equilibrium Problems
}

\author{
Dang Van Hieu ${ }^{a}$ \\ a Department of Mathematics, Vietnam National University, Hanoi \\ 334 - Nguyen Trai Street, Ha Noi, Viet Nam \\ E-mail: dv.hieu83@gmail.com
}

Received November 9, 2015; revised April 23, 2016; published online July 1, 2016

\begin{abstract}
In this paper, we introduce two parallel extragradient-proximal methods for solving split equilibrium problems. The algorithms combine the extragradient method, the proximal method and the shrinking projection method. The weak and strong convergence theorems for iterative sequences generated by the algorithms are established under widely used assumptions for equilibrium bifunctions. We also present an application to split variational inequality problems and a numerical example to illustrate the convergence of the proposed algorithms.
\end{abstract}

Keywords: equilibrium problem, split equilibrium problem, extragradient method, proximal method, parallel algorithm.

AMS Subject Classification: 90C33, 68W10, 65K10.

\section{Introduction}

Let $H_{1}, H_{2}$ be two real Hilbert spaces and $C, Q$ be two nonempty closed convex subsets of $H_{1}, H_{2}$, respectively. Let $A: H_{1} \rightarrow H_{2}$ be a bounded linear operator. Let $f: C \times C \rightarrow \Re$ and $F: Q \times Q \rightarrow \Re$ be two bifunctions with $f(x, x)=0$ for all $x \in C$ and $F(y, y)=0$ for all $y \in Q$. The split equilibrium problem (SEP) [17] is stated as follows:

$$
\left\{\begin{array}{l}
\text { Find } x^{*} \in C \text { such that } f\left(x^{*}, y\right) \geq 0, \forall y \in C, \\
\text { and } u^{*}=A x^{*} \in Q \text { solves } F\left(u^{*}, u\right) \geq 0, \forall u \in Q .
\end{array}\right.
$$

Obviously, if $F=0$ and $Q=H_{2}$ then SEP (1.1) becomes the following equilibrium problem (EP) [3].

$$
\text { Find } x^{*} \in C \text { such that } f\left(x^{*}, y\right) \geq 0, \forall y \in C \text {. }
$$

The solution set of $\mathrm{EP}(1.2)$ for the bifunction $f$ on $C$ is denoted by $\operatorname{EP}(f, C)$. A model in practice which comes to establish SEP (1.1) is the model in intensitymodulated radiation therapy (IMRT) treatment planning [6]. A mentioned 
archetypal model in Section 2 of [7] is the Split Inverse Problem (SIP) where there are a bounded linear operator $A$ from a space $X$ to another space $Y$ and two inverse problems IP1 and IP2 installed in $X$ and $Y$, respectively. The SIP is stated as follows:

$$
\left\{\begin{array}{l}
\text { find a point } x^{*} \in X \text { that solves IP1 such that } \\
\text { the point } y^{*}=A x^{*} \in Y \text { that solves IP2. }
\end{array}\right.
$$

Many models of inverse problems in this framework can be predicted by choosing different inverse problems for IP1 and IP2. Two most notable examples are the split convex feasibility problem (SCFP) and the split optimization problem (SOP) in which IP1 and IP2 are two convex feasibility problems (CFP) or two constrained optimization problems (COP), see [9,24]. The idea of modelling for SIP (1.3) also originates from CFPs and COPs which have been used to model for many inverse problems in various areas of mathematics, physical sciences and significant real world inverse problems $[4,5,7]$. It is natural to study SIP (1.3) for other inverse models for IP1 and IP2. Censor et al. [7] introduced the split variational inequality problem (SVIP) in which both IP1 and IP2 are variational inequality problems (VIP). Moudafi $[24,25,26]$ introduced and studied the notions of split equality problem and split variational inclusion problem.

It is also well known that EP (1.2) is a generalization of many mathematical models [3] involving VIPs, COPs, CFPs and fixed point problems (FPP). The EP is very important in the field of applied mathematics. Moreover, in recent years, the problem of finding a common solution to equilibrium problems (CSEP) has been widely and intensively studied by many authors. The following is a simple model for CSEP which comes from Nash-Cournot oligopolistic equilibrium model [15]. Suppose that there are $n$ companies that produce a commodity. Let $x$ denote the vector whose entry $x_{j}$ stands for the quantity of the commodity producing by company $j$ and $K_{j}$ be the strategy set of company $j$. Then the strategy set of the model is $K:=K_{1} \times \times \ldots \times K_{n}$. Assume that the price $p_{i}(s)$ is a decreasing affine function of $s$ with $s=\sum_{j=1}^{n} x_{j}$, i.e.,

$$
p_{i}(s)=\alpha_{i}-\beta_{i} s
$$

where $\alpha_{i}>0, \beta_{i}>0$. Then the profit made by company $j$ is given by

$$
f_{j}(x)=p_{j}(s) x_{j}-c_{j}\left(x_{j}\right),
$$

where $c_{j}\left(x_{j}\right)$ is the tax for generating $x_{j}$. In fact, each company seeks to maximize its profit by choosing the corresponding production level under the presumption that the production of the other companies is a parametric input. A commonly used approach to this model is based upon the famous Nash equilibrium concept. We recall that a point $x^{*} \in K=K_{1} \times K_{2} \times \cdots \times K_{n}$ is an equilibrium point of the model if

$$
f_{j}\left(x^{*}\right) \geq f_{j}\left(x^{*}\left[x_{j}\right]\right) \forall x_{j} \in K_{j}, \forall j=1,2, \ldots, n,
$$

where the vector $x^{*}\left[x_{j}\right]$ stands for the vector obtained from $x^{*}$ by replacing $x_{j}^{*}$ with $x_{j}$. Define the bifunction $f$ by

$$
f(x, y):=\psi(x, y)-\psi(x, x)
$$


with $\psi(x, y):=-\sum_{j=1}^{n} f_{j}\left(x\left[y_{j}\right]\right)$. The problem of finding a Nash equilibrium point of the model can be formulated as: Find $x^{*} \in K$ such that

$$
f\left(x^{*}, y\right) \geq 0 \forall y \in K .
$$

Note that the convex assumption on $c_{j}$ implies that the bifunction $f$ is monotone on $K$. In practice each company has to pay a fee $g_{j}\left(x_{j}\right)$ depending on its production level $x_{j}$. We suppose that both tax and fee functions are convex for every $j$. The convexity assumption means that the tax and fee for producing a unit are increasing as the quantity of the production gets larger. The problem now is to find an equilibrium point with minimum fee, i.e., the solution $x^{*}$ of (EP1) also solves the following equilibrium problem: Find $x^{*} \in K$ such that

$$
F\left(x^{*}, y\right) \geq 0, \forall y \in K,
$$

where $F(x, y)=g(y)-g(x)$ and $g(x)=\sum_{j=1}^{n} g_{j}\left(x_{j}\right)$. We see that the problem of finding a common solution of (EP1) and (EP2) is on a same feasible set $K$ and on a same space $\Re^{n}$. As a generalization, when the feasible sets of (EP1) and (EP2) are different in a same space, or in more general, (EP1) and (EP2) are in two different spaces which originates from the model of SIP (1.3), i.e., a split equilibrium problem (SEP) should enable us to split equilibrium solutions between two different subsets of spaces in which the image of a solution point of one problem, under a given bounded linear operator, is a solution point of another problem.

Moreover, the multi-objective split optimization problem (MSOP) has been considered by some authors in recent years, for examples, in $[9,24]$ and the references therein. This problem is stated as follows:

$$
\left\{\begin{array}{l}
\text { Find } x^{*} \in C \subset H_{1} \text { that solves } \min \left\{g_{i}(x): x \in C\right\}, i=1, \ldots, N \\
\text { such that } \\
u^{*}=A x^{*} \subset Q \subset H_{2} \text { solves } \min \left\{h_{j}(u): u \in Q\right\}, j=1, \ldots, M,
\end{array}\right.
$$

where $g_{i}, h_{j}$ are convex objective functions on $C$ and $Q$, respectively. If the functions $g_{i}$ and $h_{j}$ are differentiable for all $i, j$ then MSOP (1.4) can be solved by many different methods or reformulated equivalently to the multiple set SVIP [7, Section 6.1] for derivative operators $\nabla g_{i}$ and $\nabla h_{j}$. However, if $g_{i}$ or/and $h_{j}$ are only convex and not differentiable for some $i, j$ then, by setting $f_{i}(x, y)=g_{i}(y)-g_{i}(x)$ and $F_{j}(u, v)=h_{j}(v)-h_{j}(u)$, MSOP (1.4) is equivalent to the SEP considered in this paper.

The interest is to cover many situations and some practical models are promosing in the future, for examples, decomposition methods for PDEs [2], game theory and equilibrium models [15] and intensity-dodulated radiation therapy [6]. Recently, SEP (1.1) and its special cases have been recieved a lot of attention by many authors and some methods for solving them can be found, for instance, in $[8,11,12,13,14,17,19,20,22,24,25,26,30,32]$. Almost proposed methods for SEPs based on the proximal method [21] which consists of solving a regularized equilibrium problem, i.e., at current iteration, given $x_{n}$, the next iterate $x_{n+1}$ solves the following problem;

$$
\text { Find } x \in C \text { such that } f(x, y)+\frac{1}{r_{n}}\left\langle y-x, x-x_{n}\right\rangle \geq 0, \forall y \in C,
$$


or $x_{n+1}=T_{r_{n}}^{f}\left(x_{n}\right)$ where $T_{r_{n}}^{f}$ is the resolvent of the bifunction $f$ and $r_{n}>0$, see [10]. In 2012, He [17] used the proximal method and proposed the following algorithm;

$$
\left\{\begin{array}{l}
f_{i}\left(u_{n}^{i}, y\right)+\frac{1}{r_{n}}\left\langle y-u_{n}^{i}, u_{n}^{i}-x_{n}\right\rangle \geq 0, \forall y \in C, i=1, \ldots, N, \\
\tau_{n}=\frac{u_{n}^{1}+\ldots+u_{n}^{N}}{N} \\
F\left(w_{n}, z\right)+\frac{1}{r_{n}}\left\langle z-w_{n}, w_{n}-\tau_{n}\right\rangle \geq 0, \forall z \in Q, \\
x_{n+1}=P_{C}\left(\tau_{n}+\mu A^{*}\left(w_{n}-A \tau_{n}\right)\right)
\end{array}\right.
$$

for finding an element in $\Omega=\left\{p \in \cap_{i=1}^{N} E P\left(f_{i}, C\right): A p \in E P(F, Q)\right\}$. Under the assumption of the monotonicity of $f_{i}: C \times C \rightarrow \Re, F: Q \times Q \rightarrow \Re$ and suitable conditions on the parameters $r_{n}, \mu$, the author proved that $\left\{u_{n}^{i}\right\},\left\{x_{n}\right\}$ converge weakly to some point in $\Omega$.

Very recently, for finding a common solution of a system of equilibrium problems for pseudomonotone monotone and Lipschitz-type continuous bifunctions $\left\{f_{i}\right\}_{i=1}^{N}$, the authors in [18] have proposed the following parallel hybrid extragradient algorithm;

$$
\left\{\begin{array}{l}
y_{n}^{i}=\operatorname{argmin}\left\{\lambda f_{i}\left(x_{n}, y\right)+\frac{1}{2}\left\|x_{n}-y\right\|^{2}: y \in C\right\} \\
z_{n}^{i}=\operatorname{argmin}\left\{\lambda f_{i}\left(y_{n}^{i}, y\right)+\frac{1}{2}\left\|x_{n}-y\right\|^{2}: y \in C\right\} \\
\bar{z}_{n}=\operatorname{argmax}\left\{\left\|z_{n}^{i}-x_{n}\right\|: i=1, \ldots, N\right\} \\
C_{n}=\left\{v \in C:\left\|\bar{z}_{n}-v\right\| \leq\left\|x_{n}-v\right\|\right\} \\
Q_{n}=\left\{v \in C:\left\langle x_{0}-x_{n}, v-x_{n}\right\rangle \leq 0\right\} \\
x_{n+1}=P_{C_{n}} \cap Q_{n} x_{0}, n \geq 0 .
\end{array}\right.
$$

It has been proved that $\left\{x_{n}\right\},\left\{y_{n}^{i}\right\},\left\{z_{n}^{i}\right\}$ converge strongly to the projection of the starting point $x_{0}$ onto the solution set $F:=\cap_{i=1}^{N} E P\left(f_{i}, C\right)$ under certain conditions on the parameter $\lambda$. The advantages of the extragradient method are that it is used for the class of pseudomonotone bifunctions and two optimization programs are solved at each iteration which seems to be numerically easier than non-linear inequality (1.5) in the proximal method, see for instance $[28,31,33]$ and the references therein.

Motivated and inspired by the recent works $[7,9,13,19,20]$ and the results above, we consider SIP (1.3) in Hilbert spaces $H_{1}$ and $H_{2}$ in which IP1 and IP2 are CSEPs. We propose two parallel extragradient-proximal methods for SEPs for a finite family of bifunctions $\left\{f_{i}\right\}_{i=1}^{N}: C \times C \rightarrow \Re$ in $H_{1}$ and a system of bifunctions $\left\{F_{j}\right\}_{j=1}^{M}: Q \times Q \rightarrow \Re$ in $H_{2}$. We first use the extragradient method for pseudomonotone EPs in $H_{1}$ and the proximal method for monotone EPs in $H_{2}$ to design the weak convergence algorithm. In order to obtain the strong convergence, we combine the first one with the shrinking projection method in the second algorithm. Under widely used assumptions for bifunctions, the convergence theorems are proved.

The paper is organized as follows: In Section 2, we collect some definitions and preliminary results for the further use. Section 3 deals with proposing and analyzing the convergence of the algorithms. An application to SVIPs is mentioned in Section 4. Section 5 presents a numerical example to demonstrate the convergence of the algorithms. 


\section{Preliminaries}

Let $C$ be a nonempty closed convex subset of a real Hilbert space $H$ with the inner product $\langle.,$.$\rangle and the induced norm \|$.$\| . Let \left\{x_{n}\right\}$ be a sequence in $H$ and $x \in H$, we write $x_{n} \rightarrow x\left(x_{n} \rightarrow x\right)$ to stand for the strong (weak) convergence of $\left\{x_{n}\right\}$ to $x$. We begin with some concepts of the monotonicity of a bifunction.

Definition 1. [3,27] A bifunction $f: C \times C \rightarrow \Re$ is said to be

i. Strongly monotone on $C$ if there exists a constant $\gamma>0$ such that

$$
f(x, y)+f(y, x) \leq-\gamma\|x-y\|^{2}, \forall x, y \in C
$$

ii. Monotone on $C$ if

$$
f(x, y)+f(y, x) \leq 0, \forall x, y \in C
$$

iii. Pseudomonotone on $C$ if

$$
f(x, y) \geq 0 \Longrightarrow f(y, x) \leq 0, \forall x, y \in C
$$

iv. Lipschitz-type continuous on $C$ if there exist two positive constants $c_{1}, c_{2}$ such that

$$
f(x, y)+f(y, z) \geq f(x, z)-c_{1}\|x-y\|^{2}-c_{2}\|y-z\|^{2}, \forall x, y, z \in C .
$$

From the definitions above, it is clear that a strongly monotone bifunction is monotone and a monotone bifunction is pseudomonotone, i.e., $i . \Longrightarrow i i . \Longrightarrow i i i$.

For solving SEP (1.1), we set the following conditions for the bifunctions $f: C \times C \rightarrow \Re$ and $F: Q \times Q \rightarrow \Re$. Firstly, for establishing a weakly convergence algorithm, we assume that $f$ satisfies the following condition.

\section{Condition 1}

(A1) $f$ is pseudomonotone on $C$ and $f(x, x)=0$ for all $x \in C$;

(A2) $f$ is Lipschitz-type continuous on $C$ with the constants $c_{1}, c_{2}$;

(A3) $f(., y)$ is weakly sequencially upper semicontinuous on $C$ with every fixed $y \in C$, i.e., $\lim _{n \rightarrow \infty} \sup _{n \rightarrow \infty} f\left(x_{n}, y\right) \leq f(x, y)$ for each sequence $\left\{x_{n}\right\} \subset C$ converging weakly to $x$.

(A4) $f(x,$.$) is convex and subdifferentiable on C$ for every fixed $x \in C$.

Next, for obtaining a strongly convergence algorithm, we replace the assumption (A3) in Condition 1 by the weaker one (A3a) below, i.e., the bifunction $f$ satisfies the following condition.

Condition 1a The assumptions (A1), (A2), (A4) in Condition 1 hold, and 
(A3a) $f(., y)$ is sequencially upper semicontinuous on $C$ with every fixed $y \in C$, i.e., $\lim \sup _{n \rightarrow \infty} f\left(x_{n}, y\right) \leq f(x, y)$ for each sequence $\left\{x_{n}\right\} \subset C$ converging strongly to $x$.

Throughout this paper, the bifunction $F$ satisfies the following condition.

\section{Condition 2}

$(\overline{\mathrm{A}} 1) \quad F$ is monotone on $C$ and $F(x, x)=0$ for all $x \in C$;

$(\overline{\mathrm{A}} 2)$ For all $x, y, z \in C$,

$$
\lim _{t \rightarrow 0^{+}} \sup F(t z+(1-t) x, y) \leq F(x, y)
$$

$(\overline{\mathrm{A}} 3) \quad$ For all $x \in C, F(x,$.$) is convex and lower semicontinuous.$

Hypothesis (A2) was introduced by Mastroeni [23] to prove the convergence of the auxiliary principle method for solving an equilibrium problem. If $U: H \rightarrow H$ is a $L$ - Lipschitz continuous (nonlinear) operator then the bifunction $f(x, y)=\langle U(x), y-x\rangle$ satisfies hypothesis (A2) with $c_{1}=c_{2}=L / 2$. Hypothesis (A3) was used by the authors in [33]. If $U$ is compact and linear then the bifunction $f$ satisfies condition (A3), in addition, if $U$ is self-adjoint and positive semidefinite then $f$ satisfies Condition 1 , for example, $U$ is a linear integral operator with the kernel being symmetric and continuous in $L^{2}[a, b]$. Condition 1a holds under the assumption that $U$ is $L$ - Lipschitz continuous and pseudomonotone (not necessarily linear).

In Euclidean space $\Re^{n}$, the bifunction $f(x, y)=\langle P x+Q y+q, y-x\rangle$ which comes from Nash - Cournot equilibrium model [31] satisfies both Condition 1 and Condition 1a with $c_{1}=c_{2}=\|Q-P\| / 2$, where $P, Q$ are two matrices of order $n$ such that $Q$ is symmetric positive semidefinite and $Q-P$ is negative semidefinite, and $q \in \Re^{n}$. Several examples for bifunctions satisfy Condition 2 are provided in [10].

The following results concern with the monotone bifunction $F$.

Lemma 1. [10, Lemma 2.12] Let $C$ be a nonempty, closed and convex subset of a Hilbert space $H, F$ be a bifunction from $C \times C$ to $\Re$ satisfying Condition 2 and let $r>0, x \in H$. Then, there exists $z \in C$ such that

$$
F(z, y)+\frac{1}{r}\langle y-z, z-x\rangle \geq 0, \quad \forall y \in C .
$$

Lemma 2. [10, Lemma 2.12] Let $C$ be a nonempty, closed and convex subset of a Hilbert space $H, F$ be a bifunction from $C \times C$ to $\Re$ satisfying Condition 2. For all $r>0$ and $x \in H$, define the mapping

$$
T_{r}^{F} x=\left\{z \in C: F(z, y)+\frac{1}{r}\langle y-z, z-x\rangle \geq 0, \quad \forall y \in C\right\} .
$$

Then the followings hold:

(B1) $T_{r}^{F}$ is single-valued; 
(B2) $T_{r}^{F}$ is a firmly nonexpansive, i.e., for all $x, y \in H$

$$
\left\|T_{r}^{F} x-T_{r}^{F} y\right\|^{2} \leq\left\langle T_{r}^{F} x-T_{r}^{F} y, x-y\right\rangle ;
$$

(B3) Fix $\left(T_{r}^{F}\right)=\operatorname{EP}(F, C)$, where Fix $\left(T_{r}^{F}\right)$ is the fixed point set of $T_{r}^{F}$;

(B4) $E P(F, C)$ is closed and convex.

Lemma 3. [17, Lemma 2.5] For $r, s>0$ and $x, y \in H$. Under the assumptions of Lemma 2, then

$$
\left\|T_{r}^{F}(x)-T_{s}^{F}(y)\right\| \leq\|x-y\|+\frac{|s-r|}{s}\left\|T_{s}^{F}(y)-y\right\| .
$$

The metric projection $P_{C}: H \rightarrow C$ is defined by $P_{C} x=\underset{y \in C}{\arg \min }\{\|y-x\|\}$. It is well-known that $P_{C}$ has the following characteristic properties, see [16] for more details.

Lemma 4. Let $P_{C}: H \rightarrow C$ be the metric projection from $H$ onto $C$. Then

i. For all $x \in C, y \in H$,

$$
\left\|x-P_{C} y\right\|^{2}+\left\|P_{C} y-y\right\|^{2} \leq\|x-y\|^{2} .
$$

ii. $z=P_{C} x$ if and only if $\langle x-z, z-y\rangle \geq 0, \forall y \in C$.

Any Hilbert space satisfies Opial's condition [29], i.e., if $\left\{x_{n}\right\} \subset H$ converges weakly to $x$ then

$$
\lim \inf _{n \rightarrow \infty}\left\|x_{n}-x\right\|<\lim \inf _{n \rightarrow \infty}\left\|x_{n}-y\right\|, \forall y \in H, y \neq x .
$$

\section{Main results}

In this section, we present our algorithms and prove their convergence. Without loss of generality, we assume that all bifunctions $f_{i}: C \times C \rightarrow \Re$ satisfy the Lipschitz-type continuous condition with same constants $c_{1}, c_{2}$. Indeed, if $f_{i}$ is Lipschitz-type continuous with two constants $c_{1}^{i}, c_{2}^{i}$ then we set $c_{1}=$ $\max \left\{c_{1}^{i}: i=1, \ldots, N\right\}$ and $c_{2}=\max \left\{c_{2}^{i}: i=1, \ldots, N\right\}$. From the definition of the Lipschitz-type continuity, $f_{i}$ is also Lipschitz-type continuous with the constants $c_{1}, c_{2}$. We denote the solution set of SEP for $\left\{f_{i}\right\}_{i=1}^{N}$ and $\left\{F_{j}\right\}_{j=1}^{M}$ by

$$
\Omega=\left\{x^{*} \in \cap_{i=1}^{N} E P\left(f_{i}, C\right): A x^{*} \in \cap_{j=1}^{M} E P\left(F_{j}, Q\right)\right\} .
$$

It is easy to show that if $f_{i}$ satisfies Condition 1 or Condition 1 a then the solution set $\operatorname{EP}\left(f_{i}, C\right)$ is closed and convex, see for instance [31]. Moreover, from Lemma 2 (B4), under Condition 2 the set of solutions $E P\left(F_{j}, Q\right)$ is also closed and convex. Since the operator $A$ is linear and bounded, $\Omega$ is closed and convex. In this paper, we assume that $\Omega$ is nonempty. We start with the following algorithm. 
Algorithm 1. (Parallel extragradient-proximal method for SEPs)

Initialization. Choose $x_{0} \in C$. The control parameters $\lambda, \mu, r_{n}$ satisfy the following conditions

$$
0<\lambda<\min \left\{\frac{1}{2 c_{1}}, \frac{1}{2 c_{2}}\right\}, r_{n} \geq d>0,0<\mu<\frac{2}{\|A\|^{2}} .
$$

Step 1. Solve $2 N$ strongly convex optimization programs in parallel

$$
\left\{\begin{array}{l}
y_{n}^{i}=\arg \min \left\{\lambda f_{i}\left(x_{n}, y\right)+\frac{1}{2}\left\|y-x_{n}\right\|^{2}: y \in C\right\}, i=1, \ldots, N \\
z_{n}^{i}=\arg \min \left\{\lambda f_{i}\left(y_{n}^{i}, y\right)+\frac{1}{2}\left\|y-x_{n}\right\|^{2}: y \in C\right\}, i=1, \ldots, N
\end{array}\right.
$$

Step 2. Find among $z_{n}^{i}$ the furthest element from $x_{n}$, i.e.,

$$
\bar{z}_{n}=\arg \max \left\{\left\|z_{n}^{i}-x_{n}\right\|: i=1, \ldots, N\right\} .
$$

Step 3. Solve $M$ regularized equilibrium programs in parallel

$$
w_{n}^{j}=T_{r_{n}}^{F_{j}}\left(A \bar{z}_{n}\right), \quad j=1, \ldots, M .
$$

Step 4. Find among $w_{n}^{j}$ the furthest element from $A \bar{z}_{n}$, i.e.,

$$
\bar{w}_{n}=\arg \max \left\{\left\|w_{n}^{j}-A \bar{z}_{n}\right\|: j=1, \ldots, M\right\} .
$$

Step 5. Compute $x_{n+1}=P_{C}\left(\bar{z}_{n}+\mu A^{*}\left(\bar{w}_{n}-A \bar{z}_{n}\right)\right)$. Set $n=n+1$ and go back Step 1.

We need the following lemma to prove the convergence of Algorithm 1.

Lemma 5. [1, Lemma 3.1] Suppose that $x^{*} \in \cap_{i=1}^{N} E P\left(f_{i}, C\right)$ and $\left\{x_{n}\right\},\left\{y_{n}^{i}\right\}$, $\left\{z_{n}^{i}\right\}$ are the sequences generated by Algorithm 1. Then

$$
\begin{aligned}
& \text { i. } \lambda\left(f_{i}\left(x_{n}, y\right)-f_{i}\left(x_{n}, y_{n}^{i}\right)\right) \geq\left\langle y_{n}^{i}-x_{n}, y_{n}^{i}-y\right\rangle, \forall y \in C . \\
& \text { ii. }\left\|z_{n}^{i}-x^{*}\right\|^{2} \leq\left\|x_{n}-x^{*}\right\|^{2}-\left(1-2 \lambda c_{1}\right)\left\|y_{n}^{i}-x_{n}\right\|^{2}-\left(1-2 \lambda c_{2}\right)\left\|y_{n}^{i}-z_{n}^{i}\right\|^{2} .
\end{aligned}
$$

Theorem 1 [Weak convergence theorem]. Let $C, Q$ be two nonempty closed convex subsets of two real Hilbert spaces $H_{1}$ and $H_{2}$, respectively. Let $\left\{f_{i}\right\}_{i=1}^{N}: C \times C \rightarrow \Re$ be a finite family of bifunctions satisfying Condition 1 and $\left\{F_{j}\right\}_{j=1}^{M}: Q \times Q \rightarrow \Re$ be a finite family of bifunctions satisfying Condition 2. Let $A: H_{1} \rightarrow H_{2}$ be a bounded linear operator with the adjoint $A^{*}$. In addition the solution set $\Omega$ is nonempty. Then, the sequences $\left\{x_{n}\right\}$, $\left\{y_{n}^{i}\right\},\left\{z_{n}^{i}\right\}, i=1, \ldots, N$ generated by Algorithm 1 converge weakly to some point $p \in \cap_{i=1}^{N} E P\left(f_{i}, C\right)$ and $\left\{w_{n}^{j}\right\}, j=1, \ldots, M$ converge weakly to $A p \in$ $\cap_{j=1}^{M} \operatorname{EP}\left(F_{j}, Q\right)$.

Proof. We divide the proof of Theorem 1 into three claims.

Claim 1. There exists the limit of the sequence $\left\{\left\|x_{n}-x^{*}\right\|\right\}$ for all $x^{*} \in \Omega$. The proof of Claim 1. From Lemma 5.ii. and the hypothesis of $\lambda$, we have $\left\|z_{n}^{i}-x^{*}\right\| \leq\left\|x_{n}-x^{*}\right\|$ for all $x^{*} \in \Omega$. Thus,

$$
\left\|\bar{z}_{n}-x^{*}\right\| \leq\left\|x_{n}-x^{*}\right\| .
$$


Suppose $j_{n} \in\{1, \ldots, M\}$ such that $\bar{w}_{n}=w_{n}^{j_{n}}$. From Lemma 2(B2), we have

$$
\begin{aligned}
\left\|\bar{w}_{n}-A x^{*}\right\|^{2} & =\left\|T_{r_{n}}^{F_{j_{n}}}\left(A \bar{z}_{n}\right)-T_{r_{n}}^{F_{j_{n}}}\left(A x^{*}\right)\right\|^{2} \\
& \leq\left\langle T_{r_{n}}^{F_{j_{n}}}\left(A \bar{z}_{n}\right)-T_{r_{n}}^{F_{j_{n}}}\left(A x^{*}\right), A \bar{z}_{n}-A x^{*}\right\rangle \\
& =\left\langle\bar{w}_{n}-A x^{*}, A \bar{z}_{n}-A x^{*}\right\rangle \\
& =\frac{1}{2}\left\{\left\|\bar{w}_{n}-A x^{*}\right\|^{2}+\left\|A \bar{z}_{n}-A x^{*}\right\|^{2}-\left\|\bar{w}_{n}-A \bar{z}_{n}\right\|^{2}\right\} .
\end{aligned}
$$

Thus,

$$
\left\|\bar{w}_{n}-A x^{*}\right\|^{2} \leq\left\|A \bar{z}_{n}-A x^{*}\right\|^{2}-\left\|\bar{w}_{n}-A \bar{z}_{n}\right\|^{2}
$$

or

$$
\left\|\bar{w}_{n}-A x^{*}\right\|^{2}-\left\|A \bar{z}_{n}-A x^{*}\right\|^{2} \leq-\left\|\bar{w}_{n}-A \bar{z}_{n}\right\|^{2} .
$$

This together with the following fact

$$
\left\langle A\left(\bar{z}_{n}-x^{*}\right), \bar{w}_{n}-A \bar{z}_{n}\right\rangle=\frac{1}{2}\left\{\left\|\bar{w}_{n}-A x^{*}\right\|^{2}-\left\|A \bar{z}_{n}-A x^{*}\right\|^{2}-\left\|\bar{w}_{n}-A \bar{z}_{n}\right\|^{2}\right\}
$$

implies that

$$
\left\langle A\left(\bar{z}_{n}-x^{*}\right), \bar{w}_{n}-A \bar{z}_{n}\right\rangle \leq-\left\|\bar{w}_{n}-A \bar{z}_{n}\right\|^{2} .
$$

Thus, from the definition of $x_{n+1}$ and the nonexpansiveness of the projection,

$$
\begin{aligned}
& \left\|x_{n+1}-x^{*}\right\|^{2}=\left\|P_{C}\left(\bar{z}_{n}+\mu A^{*}\left(\bar{w}_{n}-A \bar{z}_{n}\right)\right)-P_{C} x^{*}\right\|^{2} \\
& \quad \leq\left\|\bar{z}_{n}-x^{*}+\mu A^{*}\left(\bar{w}_{n}-A \bar{z}_{n}\right)\right\|^{2} \\
& \quad=\left\|\bar{z}_{n}-x^{*}\right\|^{2}+\mu^{2}\left\|A^{*}\left(\bar{w}_{n}-A \bar{z}_{n}\right)\right\|^{2}+2 \mu\left\langle\bar{z}_{n}-x^{*}, A^{*}\left(\bar{w}_{n}-A \bar{z}_{n}\right)\right\rangle \\
& \quad \leq\left\|\bar{z}_{n}-x^{*}\right\|^{2}+\mu^{2}\left\|A^{*}\right\|^{2}\left\|\bar{w}_{n}-A \bar{z}_{n}\right\|^{2}+2 \mu\left\langle A\left(\bar{z}_{n}-x^{*}\right), \bar{w}_{n}-A \bar{z}_{n}\right\rangle \\
& \quad \leq\left\|\bar{z}_{n}-x^{*}\right\|^{2}+\mu^{2}\left\|A^{*}\right\|^{2}\left\|\bar{w}_{n}-A \bar{z}_{n}\right\|^{2}-2 \mu\left\|\bar{w}_{n}-A \bar{z}_{n}\right\|^{2} \\
& \quad \leq\left\|\bar{z}_{n}-x^{*}\right\|^{2}-\mu\left(2-\mu\left\|A^{*}\right\|^{2}\right)\left\|\bar{w}_{n}-A \bar{z}_{n}\right\|^{2} \\
& \quad \leq\left\|\bar{z}_{n}-x^{*}\right\|^{2}
\end{aligned}
$$

in which the last inequality is followed from the assumption of $\mu$. From the relations (3.1) and (3.3),

$$
0 \leq\left\|x_{n+1}-x^{*}\right\| \leq\left\|\bar{z}_{n}-x^{*}\right\| \leq\left\|x_{n}-x^{*}\right\|, \forall x^{*} \in \Omega .
$$

Therefore, the sequence $\left\{\left\|x_{n+1}-x^{*}\right\|\right\}$ is decreasing and so there exist the limits

$$
\lim _{n \rightarrow \infty}\left\|x_{n}-x^{*}\right\|=\lim _{n \rightarrow \infty}\left\|\bar{z}_{n}-x^{*}\right\|=p\left(x^{*}\right), \forall x^{*} \in \Omega .
$$

Claim 2. $\lim _{n \rightarrow \infty}\left\|z_{n}^{i}-x_{n}\right\|=\lim _{n \rightarrow \infty}\left\|y_{n}^{i}-x_{n}\right\|=\lim _{n \rightarrow \infty}\left\|w_{n}^{j}-A \bar{z}_{n}\right\|=0$.

The proof of Claim 2. Suppose that $i_{n}$ is the index in $\{1, \ldots, N\}$ such that $\bar{z}_{n}=z_{n}^{i_{n}}$. From Lemma 5.ii. with $i=i_{n}$,

$$
\left\|\bar{z}_{n}-x^{*}\right\|^{2} \leq\left\|x_{n}-x^{*}\right\|^{2}-\left(1-2 \lambda c_{1}\right)\left\|y_{n}^{i_{n}}-x_{n}\right\|^{2}-\left(1-2 \lambda c_{2}\right)\left\|y_{n}^{i_{n}}-\bar{z}_{n}\right\|^{2} .
$$

Thus,

$$
\left(1-2 \lambda c_{1}\right)\left\|y_{n}^{i_{n}}-x_{n}\right\|^{2}+\left(1-2 \lambda c_{2}\right)\left\|y_{n}^{i_{n}}-\bar{z}_{n}\right\|^{2} \leq\left\|x_{n}-x^{*}\right\|^{2}-\left\|\bar{z}_{n}-x^{*}\right\|^{2} .
$$


This together with (3.4) and the hypothesis of $\lambda$ implies that

$$
\lim _{n \rightarrow \infty}\left\|y_{n}^{i_{n}}-x_{n}\right\|=\lim _{n \rightarrow \infty}\left\|y_{n}^{i_{n}}-\bar{z}_{n}\right\|=0 .
$$

Thus,

$$
\lim _{n \rightarrow \infty}\left\|\bar{z}_{n}-x_{n}\right\|=0
$$

because of $\left\|\bar{z}_{n}-x_{n}\right\| \leq\left\|y_{n}^{i_{n}}-x_{n}\right\|+\left\|y_{n}^{i_{n}}-\bar{z}_{n}\right\|$. It follows from the last limit and the definition of $\bar{z}_{n}$ that

$$
\lim _{n \rightarrow \infty}\left\|z_{n}^{i}-x_{n}\right\|=0, \forall i=1, \ldots, N .
$$

From Lemma 5.ii. and the triangle inequality,

$$
\begin{aligned}
\left(1-2 \lambda c_{1}\right)\left\|y_{n}^{i}-x_{n}\right\|^{2} & \leq\left\|x_{n}-x^{*}\right\|^{2}-\left\|z_{n}^{i}-x^{*}\right\|^{2} \\
& =\left(\left\|x_{n}-x^{*}\right\|-\left\|z_{n}^{i}-x^{*}\right\|\right)\left(\left\|x_{n}-x^{*}\right\|+\left\|z_{n}^{i}-x^{*}\right\|\right) \\
& \leq\left\|x_{n}-z_{n}^{i}\right\|\left(\left\|x_{n}-x^{*}\right\|+\left\|z_{n}^{i}-x^{*}\right\|\right),
\end{aligned}
$$

which implies that

$$
\lim _{n \rightarrow \infty}\left\|y_{n}^{i}-x_{n}\right\|=0
$$

because of the relation (3.5), the hypothesis of $\lambda$ and the boundedness of $\left\{x_{n}\right\},\left\{z_{n}^{i}\right\}$. Moreover, from (3.2), we obtain

$$
\mu\left(2-\mu\left\|A^{*}\right\|^{2}\right)\left\|\bar{w}_{n}-A \bar{z}_{n}\right\|^{2} \leq\left\|\bar{z}_{n}-x^{*}\right\|^{2}-\left\|x_{n+1}-x^{*}\right\|^{2} .
$$

Passing to the limit in the last inequality as $n \rightarrow \infty$ and using the relation (3.4) and $\mu\left(2-\mu\left\|A^{*}\right\|^{2}\right)>0$, one has

$$
\lim _{n \rightarrow \infty}\left\|\bar{w}_{n}-A \bar{z}_{n}\right\|=0 .
$$

From the definition of $\bar{w}_{n}$, we obtain

$$
\lim _{n \rightarrow \infty}\left\|w_{n}^{j}-A \bar{z}_{n}\right\|=0, \forall j=1, \ldots, M .
$$

Claim 3. $x_{n}, y_{n}^{i}, z_{n}^{i} \rightarrow p \in \cap_{i=1}^{N} E P\left(f_{i}, C\right)$ and $w_{n}^{j} \rightarrow A p \in \cap_{j=1}^{M} E P\left(F_{j}, Q\right)$. The proof of Claim 3. Since $\left\{x_{n}\right\}$ is bounded, there exists a subsequence $\left\{x_{m}\right\}$ of $\left\{x_{n}\right\}$ which converges weakly to $p$. Since $C$ is convex, $C$ is weakly closed, and so $p \in C$. Thus, $y_{m}^{i} \rightarrow p, z_{m}^{i} \rightarrow p$ and $A \bar{z}_{m} \rightarrow A p, w_{m}^{j} \rightarrow A p$ because of the relations $(3.5),(3.6),(3.7)$ and (3.8). It follows from Lemma 5.i. that

$$
\lambda\left(f_{i}\left(x_{m}, y\right)-f_{i}\left(x_{m}, y_{m}^{i}\right)\right) \geq\left\langle y_{m}^{i}-x_{m}, y_{m}^{i}-y\right\rangle, \forall y \in C .
$$

Substituting $y=z_{m}^{i} \in C$ into the last inequality, we obtain

$$
\lambda\left(f_{i}\left(x_{m}, z_{m}^{i}\right)-f_{i}\left(x_{m}, y_{m}^{i}\right)\right) \geq\left\langle y_{m}^{i}-x_{m}, y_{m}^{i}-z_{m}^{i}\right\rangle .
$$

From the Lipschitz-type continuity of $f_{i}$ and the relation (3.9), we have

$$
\begin{gathered}
\lambda f_{i}\left(y_{m}^{i}, z_{m}^{i}\right) \geq \lambda\left(f_{i}\left(x_{m}, z_{m}^{i}\right)-f_{i}\left(x_{m}, y_{m}^{i}\right)\right)-c_{1} \lambda\left\|y_{m}^{i}-x_{m}\right\|^{2}-c_{2} \lambda\left\|z_{m}^{i}-y_{m}^{i}\right\|^{2} \\
\geq\left\langle y_{m}^{i}-x_{m}, y_{m}^{i}-z_{m}^{i}\right\rangle-c_{1} \lambda\left\|y_{m}^{i}-x_{m}\right\|^{2}-c_{2} \lambda\left\|z_{m}^{i}-y_{m}^{i}\right\|^{2} .
\end{gathered}
$$


Similarly to Lemma 5.i., from the definition of $z_{m}^{i}$, we obtain

$$
\lambda\left(f_{i}\left(y_{m}^{i}, y\right)-f_{i}\left(y_{m}^{i}, z_{m}^{i}\right)\right) \geq\left\langle z_{m}^{i}-x_{m}, z_{m}^{i}-y\right\rangle, \forall y \in C .
$$

Thus,

$$
\lambda f_{i}\left(y_{m}^{i}, y\right) \geq \lambda f_{i}\left(y_{m}^{i}, z_{m}^{i}\right)+\left\langle z_{m}^{i}-x_{m}, z_{m}^{i}-y\right\rangle, \forall y \in C .
$$

Combining the relations (3.10) and (3.11), we obtain

$$
\begin{aligned}
\lambda f_{i}\left(y_{m}^{i}, y\right) \geq & \left\langle y_{m}^{i}-x_{m}, y_{m}^{i}-z_{m}^{i}\right\rangle-c_{1} \lambda\left\|y_{m}^{i}-x_{m}\right\|^{2} \\
& -c_{2} \lambda\left\|z_{m}^{i}-y_{m}^{i}\right\|^{2}+\left\langle z_{m}^{i}-x_{m}, z_{m}^{i}-y\right\rangle .
\end{aligned}
$$

From Claim 2 and the triangle inequality, we also have $\left\|y_{m}^{i}-z_{m}^{i}\right\| \rightarrow 0$. Thus, passing to the limit in the last inequality as $m \rightarrow \infty$ and using hypothesis (A3), $\lambda>0$, the boundedness of $\left\{z_{m}^{i}\right\}$ and $y_{m}^{i} \rightarrow p$, we obtain

$$
0 \leq \lim \sup _{m \rightarrow \infty} f_{i}\left(y_{m}^{i}, y\right) \leq f_{i}(p, y), \forall y \in C, i=1, \ldots, N
$$

i.e., $p \in \cap_{i=1}^{N} E P\left(f_{i}, C\right)$. Now, we show that $A p \in \cap_{j=1}^{M} E P\left(F_{j}, Q\right)$. By Lemma 2, $\operatorname{EP}\left(F_{j}, Q\right)=F i x\left(T_{r}^{F_{j}}\right)$ for some $r>0$. Assume that $A p \notin F i x\left(T_{r}^{F_{j}}\right)$, i.e., $A p \neq T_{r}^{F_{j}}(A p)$. By Opial's condition in $H_{2}$, the relation (3.8) and Lemma 3, we have

$$
\begin{aligned}
\lim \inf _{m \rightarrow \infty}\left\|A \bar{z}_{m}-A p\right\|<\lim \inf _{m \rightarrow \infty}\left\|A \bar{z}_{m}-T_{r}^{F_{j}}(A p)\right\| \\
\quad \leq \lim \inf _{m \rightarrow \infty}\left[\left\|A \bar{z}_{m}-T_{r_{m}}^{F_{j}}\left(A \bar{z}_{m}\right)\right\|+\left\|T_{r_{m}}^{F_{j}}\left(A \bar{z}_{m}\right)-T_{r}^{F_{j}}(A p)\right\|\right] \\
\quad=\lim \inf _{m \rightarrow \infty}\left\|T_{r_{m}}^{F_{j}}\left(A \bar{z}_{m}\right)-T_{r}^{F_{j}}(A p)\right\| \\
\quad=\lim \inf _{m \rightarrow \infty}\left\|T_{r}^{F_{j}}(A p)-T_{r_{m}}^{F_{j}}\left(A \bar{z}_{m}\right)\right\| \\
\quad \leq \lim \inf _{m \rightarrow \infty}\left[\left\|A p-A \bar{z}_{m}\right\|+\frac{\left|r-r_{m}\right|}{r_{m}}\left\|T_{r_{m}}^{F_{j}}\left(A \bar{z}_{m}\right)-A \bar{z}_{m}\right\|\right] \\
\quad=\lim \inf _{m \rightarrow \infty}\left\|A p-A \bar{z}_{m}\right\| .
\end{aligned}
$$

This is a contradiction, thus $A p \in F i x\left(T_{r}^{F_{j}}\right)=\operatorname{EP}\left(F_{j}, Q\right)$, i.e., we get that $A p \in \cap_{j=1}^{M} E P\left(F_{j}, Q\right)$.

Finally, we show that the whole sequence $\left\{x_{n}\right\}$ converges weakly to $p$. Indeed, suppose that $\left\{x_{n}\right\}$ has a subsequence $\left\{x_{k}\right\}$ which converges weakly to $q \neq p$. By Opial's condition in $H_{1}$, we have

$$
\begin{aligned}
\lim \inf _{k \rightarrow \infty}\left\|x_{k}-q\right\| & <\lim \inf _{k \rightarrow \infty}\left\|x_{k}-p\right\|=\lim \inf _{m \rightarrow \infty}\left\|x_{m}-p\right\| \\
& <\lim \inf _{m \rightarrow \infty}\left\|x_{m}-q\right\|=\lim \inf _{k \rightarrow \infty}\left\|x_{k}-q\right\| .
\end{aligned}
$$

This is a contradiction. Thus, the whole sequence $\left\{x_{n}\right\}$ converges weakly to $p$. By Claim 2, $y_{n}^{i}, z_{n}^{i} \rightarrow p$ and $w_{n}^{j} \rightarrow A p$ as $n \rightarrow \infty$. Theorem 1 is proved. 
Corollary 1. Let $C, Q$ be two nonempty closed convex subsets of two real Hilbert spaces $H_{1}$ and $H_{2}$, respectively. Let $f: C \times C \rightarrow \Re$ be a bifunction satisfying Condition 1 and $F: Q \times Q \rightarrow \Re$ be a bifunction satisfying Condition 2. Let $A: H_{1} \rightarrow H_{2}$ be a bounded linear operator with the adjoint $A^{*}$. In addition the solution set $\Omega=\left\{x^{*} \in E P(f, C): A x^{*} \in E P(F, Q)\right\}$ is nonempty. Let $\left\{x_{n}\right\}$, $\left\{y_{n}\right\},\left\{z_{n}\right\}$ and $\left\{w_{n}\right\}$ be the sequences generated by the following manner: $x_{0} \in C$ and

$$
\left\{\begin{array}{l}
y_{n}=\arg \min \left\{\lambda f\left(x_{n}, y\right)+\frac{1}{2}\left\|y-x_{n}\right\|^{2}: y \in C\right\} \\
z_{n}=\arg \min \left\{\lambda f\left(y_{n}, y\right)+\frac{1}{2}\left\|y-x_{n}\right\|^{2}: y \in C\right\} \\
w_{n}=T_{r_{n}}^{F}\left(A z_{n}\right) \\
x_{n+1}=P_{C}\left(z_{n}+\mu A^{*}\left(w_{n}-A z_{n}\right)\right)
\end{array}\right.
$$

where $\lambda, r_{n}, \mu$ satisfy the conditions in Theorem 1 . Then, the sequences $\left\{x_{n}\right\}$, $\left\{y_{n}\right\},\left\{z_{n}\right\}$ converge weakly to some point $p \in E P(f, C)$ and $\left\{w_{n}\right\}$ converges weakly to $A p \in E P(F, Q)$.

In order to obtain an algorithm which provides the strong convergence, we propose the following parallel hybrid extragradient-proximal method that combines Algorithm 1 with the shrinking projection method, see for instance [30] and the references therein.

Algorithm 2. (Parallel hybrid extragradient-proximal method for SEPs)

Initialization. Choose $x_{0} \in C, C_{0}=C$, the control parameters $\lambda, r_{n}, \mu$ satisfy the following conditions

$$
0<\lambda<\min \left\{\frac{1}{2 c_{1}}, \frac{1}{2 c_{2}}\right\}, r_{n} \geq d>0,0<\mu<\frac{2}{\|A\|^{2}} .
$$

Step 1. Solve $2 N$ strongly convex optimization programs in parallel

$$
\left\{\begin{array}{l}
y_{n}^{i}=\arg \min \left\{\lambda f_{i}\left(x_{n}, y\right)+\frac{1}{2}\left\|y-x_{n}\right\|^{2}: y \in C\right\}, i=1, \ldots, N \\
z_{n}^{i}=\arg \min \left\{\lambda f_{i}\left(y_{n}^{i}, y\right)+\frac{1}{2}\left\|y-x_{n}\right\|^{2}: y \in C\right\}, i=1, \ldots, N
\end{array}\right.
$$

Step 2. Find among $z_{n}^{i}$ the furthest element from $x_{n}$, i.e.,

$$
\bar{z}_{n}=\arg \max \left\{\left\|z_{n}^{i}-x_{n}\right\|: i=1, \ldots, N\right\} .
$$

Step 3. Solve $M$ regularized equilibrium programs in parallel

$$
w_{n}^{j}=T_{r_{n}}^{F_{j}}\left(A \bar{z}_{n}\right), j=1, \ldots, M .
$$

Step 4. Find among $w_{n}^{j}$ the furthest element from $A \bar{z}_{n}$, i.e.,

$$
\bar{w}_{n}=\arg \max \left\{\left\|w_{n}^{j}-A \bar{z}_{n}\right\|: j=1, \ldots, M\right\} .
$$

Step 5. Compute $t_{n}=P_{C}\left(\bar{z}_{n}+\mu A^{*}\left(\bar{w}_{n}-A \bar{z}_{n}\right)\right)$.

Step 6. Set $C_{n+1}=\left\{v \in C_{n}:\left\|t_{n}-v\right\| \leq\left\|\bar{z}_{n}-v\right\| \leq\left\|x_{n}-v\right\|\right\}$. Compute $x_{n+1}=P_{C_{n+1}}\left(x_{0}\right)$. Set $n=n+1$ and go back Step 1 . 
We have the following result.

Theorem 2 [Strong convergence theorem]. Let $C, Q$ be two nonempty closed convex subsets of two real Hilbert spaces $H_{1}$ and $H_{2}$, respectively. Let $\left\{f_{i}\right\}_{i=1}^{N}: C \times C \rightarrow \Re$ be a finite family of bifunctions satisfying Condition 1a and $\left\{F_{j}\right\}_{j=1}^{M}: Q \times Q \rightarrow \Re$ be a finite family of bifunctions satisfying Condition 2. Let $A: H_{1} \rightarrow H_{2}$ be a bounded linear operator with the adjoint $A^{*}$. In addition the solution set $\Omega$ is nonempty. Then, the sequences $\left\{x_{n}\right\},\left\{y_{n}^{i}\right\}$, $\left\{z_{n}^{i}\right\}, i=1, \ldots, N$ generated by Algorithm 2 converge strongly to $x^{\dagger}=P_{\Omega}\left(x_{0}\right)$ and $\left\{w_{n}^{j}\right\}, j=1, \ldots, M$ converge strongly to $A x^{\dagger} \in \cap_{j=1}^{M} E P\left(F_{j}, Q\right)$.

Proof. We also divide the proof of Theorem 2 into several claims.

Claim 1. $C_{n}$ is closed convex set and $\Omega \subset C_{n}$ for all $n \geq 0$.

The proof of Claim 1. Set

$$
C_{n}^{1}=\left\{v \in H_{1}:\left\|t_{n}-v\right\| \leq\left\|\bar{z}_{n}-v\right\|\right\}, C_{n}^{2}=\left\{v \in H_{1}:\left\|\bar{z}_{n}-v\right\| \leq\left\|x_{n}-v\right\|\right\} .
$$

Then

$$
C_{n+1}=C_{n} \cap C_{n}^{1} \cap C_{n}^{2} .
$$

Note that $C_{n}^{1}, C_{n}^{2}$ are either the halfspaces or the whole space $H_{1}$ for all $n \geq 0$. Hence, they are closed and convex. Obviously, $C_{0}=C$ is closed and convex. Suppose that $C_{n}$ is closed and convex for some $n \geq 0$. Then, from (3.12), $C_{n+1}$ is also closed and convex. By the induction, $C_{n}$ is closed and convex for all $n \geq 0$. Next, we show that $\Omega \subset C_{n}$ for all $n \geq 0$. From Lemma 5.ii. and the hypothesis of $\lambda$, we have $\left\|z_{n}^{i}-x^{*}\right\| \leq\left\|x_{n}-x^{*}\right\|$ for all $x^{*} \in \Omega$. Thus,

$$
\left\|\bar{z}_{n}-x^{*}\right\| \leq\left\|x_{n}-x^{*}\right\| .
$$

By arguing similarly to Claim 1 in the proof of Theorem 1 we obtain

$$
\begin{aligned}
\left\|t_{n}-x^{*}\right\|^{2} & \leq\left\|\bar{z}_{n}-x^{*}\right\|^{2}-\mu\left(2-\mu\left\|A^{*}\right\|^{2}\right)\left\|\bar{w}_{n}-A \bar{z}_{n}\right\|^{2} \\
& \leq\left\|\bar{z}_{n}-x^{*}\right\|^{2}
\end{aligned}
$$

From (3.13) and (3.15),

$$
\left\|t_{n}-x^{*}\right\| \leq\left\|\bar{z}_{n}-x^{*}\right\| \leq\left\|x_{n}-x^{*}\right\|, \forall x^{*} \in \Omega .
$$

Thus, by the definition of $C_{n}$ and the induction, $\Omega \subset C_{n}$ for all $n \geq 0$.

Claim 2. $\left\{x_{n}\right\}$ is a Cauchy sequence and

$$
\lim _{n \rightarrow \infty} x_{n}=\lim _{n \rightarrow \infty} y_{n}^{i}=\lim _{n \rightarrow \infty} z_{n}^{i}=p, \quad \lim _{n \rightarrow \infty} w_{n}^{j}=\lim _{n \rightarrow \infty} A \bar{z}_{n}=A p .
$$

The proof of Claim 2. From $x_{n}=P_{C_{n}}\left(x_{0}\right)$ and Lemma 4.i.,

$$
\left\|x_{n}-x_{0}\right\| \leq\left\|u-x_{0}\right\|, \forall u \in C_{n} .
$$

Therefore, $\left\|x_{n}-x_{0}\right\| \leq\left\|x_{n+1}-x_{0}\right\|$ because $x_{n+1} \in C_{n+1} \subset C_{n}$. This implies that the sequence $\left\{\left\|x_{n}-x_{0}\right\|\right\}$ is non-decreasing. The inequality (3.16) with $u=x^{\dagger}:=P_{\Omega}\left(x_{0}\right) \in \Omega \subset C_{n}$ leads to

$$
\left\|x_{n}-x_{0}\right\| \leq\left\|x^{\dagger}-x_{0}\right\|
$$


Thus, the sequence $\left\{\left\|x_{n}-x_{0}\right\|\right\}$ is bounded, and so there exists the limit of $\left\{\left\|x_{n}-x_{0}\right\|\right\}$. For all $m \geq n$, from the definition of $C_{m}$, we have $x_{m} \in C_{m} \subset C_{n}$. So, from $x_{n}=P_{C_{n}}\left(x_{0}\right)$ and Lemma 4.i.,

$$
\left\|x_{n}-x_{m}\right\|^{2} \leq\left\|x_{m}-x_{0}\right\|^{2}-\left\|x_{n}-x_{0}\right\|^{2} .
$$

Passing to the limit in the last inequality as $m, n \rightarrow \infty$, we get

$$
\lim _{m, n \rightarrow \infty}\left\|x_{n}-x_{m}\right\|=0
$$

Thus, $\left\{x_{n}\right\}$ is a Cauchy sequence and

$$
\lim _{n \rightarrow \infty}\left\|x_{n}-x_{n+1}\right\|=0 .
$$

From the definition of $C_{n+1}$ and $x_{n+1} \in C_{n+1}$, we have

$$
\left\|t_{n}-x_{n+1}\right\| \leq\left\|\bar{z}_{n}-x_{n+1}\right\| \leq\left\|x_{n}-x_{n+1}\right\| .
$$

Thus, from the triangle inequality, one has

$$
\begin{aligned}
& \left\|t_{n}-x_{n}\right\| \leq\left\|t_{n}-x_{n+1}\right\|+\left\|x_{n+1}-x_{n}\right\| \leq 2\left\|x_{n}-x_{n+1}\right\|, \\
& \left\|\bar{z}_{n}-x_{n}\right\| \leq\left\|\bar{z}_{n}-x_{n+1}\right\|+\left\|x_{n+1}-x_{n}\right\| \leq 2\left\|x_{n}-x_{n+1}\right\|, \\
& \left\|\bar{z}_{n}-t_{n}\right\| \leq\left\|\bar{z}_{n}-x_{n}\right\|+\left\|x_{n}-t_{n}\right\| \leq 4\left\|x_{n}-x_{n+1}\right\| .
\end{aligned}
$$

Three last inequalities together with the relation (3.18) imply that

$$
\lim _{n \rightarrow \infty}\left\|t_{n}-x_{n}\right\|=\lim _{n \rightarrow \infty}\left\|\bar{z}_{n}-t_{n}\right\|=\lim _{n \rightarrow \infty}\left\|\bar{z}_{n}-x_{n}\right\|=0 .
$$

Hence, from the definition of $\bar{z}_{n}$, we also obtain

$$
\lim _{n \rightarrow \infty}\left\|z_{n}^{i}-x_{n}\right\|=0, \forall i=1, \ldots, N .
$$

Since $\left\{x_{n}\right\}$ is a Cauchy sequence, $x_{n} \rightarrow p$ and

$$
\lim _{n \rightarrow \infty} t_{n}=\lim _{n \rightarrow \infty} \bar{z}_{n}=\lim _{n \rightarrow \infty} z_{n}^{i}=p, \forall i=1, \ldots, N
$$

and so

$$
\lim _{n \rightarrow \infty} A \bar{z}_{n}=A p .
$$

From the relation (3.14) and the triangle inequality, we obtain

$$
\begin{gathered}
\mu\left(2-\mu\left\|A^{*}\right\|^{2}\right)\left\|\bar{w}_{n}-A \bar{z}_{n}\right\|^{2} \leq\left\|\bar{z}_{n}-x^{*}\right\|^{2}-\left\|t_{n}-x^{*}\right\|^{2} \\
=\left(\left\|\bar{z}_{n}-x^{*}\right\|-\left\|t_{n}-x^{*}\right\|\right)\left(\left\|\bar{z}_{n}-x^{*}\right\|+\left\|t_{n}-x^{*}\right\|\right) \\
\leq\left\|\bar{z}_{n}-t_{n}\right\|\left(\left\|\bar{z}_{n}-x^{*}\right\|+\left\|t_{n}-x^{*}\right\|\right) .
\end{gathered}
$$

Thus, from $\mu\left(2-\mu\left\|A^{*}\right\|^{2}\right)>0$, the boundedness of $\left\{t_{n}\right\},\left\{\bar{z}_{n}\right\}$ and (3.19) we obtain

$$
\lim _{n \rightarrow \infty}\left\|\bar{w}_{n}-A \bar{z}_{n}\right\|=0 .
$$


From the definition of $\bar{w}_{n}$, we get

$$
\lim _{n \rightarrow \infty}\left\|w_{n}^{j}-A \bar{z}_{n}\right\|=0, \forall j=1, \ldots, M
$$

which follows from (3.21) that

$$
\lim _{n \rightarrow \infty} w_{n}^{j}=A p, \forall j=1, \ldots, M
$$

From Lemma 5.ii. and the triangle inequality, we have

$$
\begin{aligned}
\left(1-2 \lambda c_{1}\right)\left\|y_{n}^{i}-x_{n}\right\|^{2} & \leq\left\|x_{n}-x^{*}\right\|^{2}-\left\|z_{n}^{i}-x^{*}\right\|^{2} \\
& =\left(\left\|x_{n}-x^{*}\right\|-\left\|z_{n}^{i}-x^{*}\right\|\right)\left(\left\|x_{n}-x^{*}\right\|+\left\|z_{n}^{i}-x^{*}\right\|\right) \\
& \leq\left\|x_{n}-z_{n}^{i}\right\|\left(\left\|x_{n}-x^{*}\right\|+\left\|z_{n}^{i}-x^{*}\right\|\right) .
\end{aligned}
$$

Thus, from the hypothesis of $\lambda$, the boundedness of $\left\{x_{n}\right\},\left\{z_{n}^{i}\right\}$ and (3.20) we obtain

$$
\lim _{n \rightarrow \infty}\left\|y_{n}^{i}-x_{n}\right\|=0
$$

Therefore, $y_{n}^{i} \rightarrow p$ as $n \rightarrow \infty$ for all $i=1, \ldots, N$.

Claim 3. $p \in \Omega$ and $p=x^{\dagger}:=P_{\Omega}\left(x_{0}\right)$.

The proof of Claim 3. By using Claim 2, the hypothesis (A3a) and arguing similarly to (3.9)-(3.11), we also obtain $p \in \cap_{i=1}^{N} E P\left(f_{i}, C\right)$. Moreover, from Lemma 3, for some $r>0$ we have

$$
\begin{aligned}
\left\|T_{r}^{F_{j}}(A p)-A p\right\| \leq & \left\|T_{r}^{F_{j}}(A p)-T_{r_{n}}^{F_{j}}\left(A \bar{z}_{n}\right)\right\|+\left\|T_{r_{n}}^{F_{j}}\left(A \bar{z}_{n}\right)-A \bar{z}_{n}\right\|+\left\|A \bar{z}_{n}-A p\right\| \\
\leq & \left\|A p-A \bar{z}_{n}\right\|+\frac{r_{n}-r}{r_{n}}\left\|T_{r_{n}}^{F_{j}}\left(A \bar{z}_{n}\right)-A \bar{z}_{n}\right\| \\
& +\left\|T_{r_{n}}^{F_{j}}\left(A \bar{z}_{n}\right)-A \bar{z}_{n}\right\|+\left\|A \bar{z}_{n}-A p\right\| \\
= & 2\left\|A p-A \bar{z}_{n}\right\|+\frac{r_{n}-r}{r_{n}}\left\|w_{n}^{j}-A \bar{z}_{n}\right\|+\left\|w_{n}^{j}-A \bar{z}_{n}\right\| \rightarrow 0,
\end{aligned}
$$

which is followed from the relations (3.21),(3.22),(3.23) and $r_{n} \geq d>0$. Thus, $T_{r}^{F_{j}}(A p)-A p=0$ or $A p$ is a fixed point of $T_{r}^{F_{j}}$. From Lemma 2, we obtain $A p \in$ $\cap_{j=1}^{M} E P\left(F_{j}, Q\right)$. Thus, $p \in \Omega$. Finally, from $(3.17),\left\|x_{n}-x_{0}\right\| \leq\left\|x^{\dagger}-x_{0}\right\|$ where $x^{\dagger}=P_{\Omega}\left(x_{0}\right)$. Taking $n \rightarrow \infty$ in this inequality, one has $\left\|p-x_{0}\right\| \leq\left\|x^{\dagger}-x_{0}\right\|$. From the definition of $x^{\dagger}, p=x^{\dagger}$. Theorem 2 is proved.

Corollary 2. Let $C, Q$ be two nonempty closed convex subsets of two real Hilbert spaces $H_{1}$ and $H_{2}$, respectively. Let $f: C \times C \rightarrow \Re$ be a bifunction satisfying Condition $1 \mathrm{a}$ and $F: Q \times Q \rightarrow \Re$ be a bifunction satisfying Condition 2. Let $A: H_{1} \rightarrow H_{2}$ be a bounded linear operator with the adjoint $A^{*}$. In addition the solution set $\Omega=\left\{x^{*} \in E P(f, C): A x^{*} \in E P(F, Q)\right\}$ is nonempty. Let $\left\{x_{n}\right\},\left\{y_{n}\right\},\left\{z_{n}\right\},\left\{t_{n}\right\}$ and $\left\{w_{n}\right\}$ be the sequences generated 
by the following manner: $x_{0} \in C, C_{0}=C$ and

$$
\left\{\begin{array}{l}
y_{n}=\arg \min \left\{\lambda f\left(x_{n}, y\right)+\frac{1}{2}\left\|y-x_{n}\right\|^{2}: y \in C\right\}, \\
z_{n}=\arg \min \left\{\lambda f\left(y_{n}, y\right)+\frac{1}{2}\left\|y-x_{n}\right\|^{2}: y \in C\right\}, \\
w_{n}=T_{r_{n}}^{F}\left(A z_{n}\right), \\
t_{n}=P_{C}\left(z_{n}+\mu A^{*}\left(w_{n}-A z_{n}\right)\right), \\
C_{n+1}=\left\{v \in C_{n}:\left\|t_{n}-v\right\| \leq\left\|\bar{z}_{n}-v\right\| \leq\left\|x_{n}-v\right\|\right\}, \\
x_{n+1}=P_{C_{n+1}}\left(x_{0}\right),
\end{array}\right.
$$

where $\lambda, r_{n}, \mu$ satisfy the conditions in Theorem 2 . Then, the sequences $\left\{x_{n}\right\}$, $\left\{y_{n}\right\},\left\{z_{n}\right\},\left\{t_{n}\right\}$ converge strongly to $x^{\dagger}=P_{\Omega}\left(x_{0}\right)$ and $\left\{w_{n}\right\}$ converges strongly to $A x^{\dagger} \in E P(F, Q)$.

\section{Application to split variational inequality problems}

In this section, we consider the following split variational inequality problem in [7, Section 6.1];

$$
\left\{\begin{array}{l}
\text { Find } x^{*} \in C \text { such that } \\
\left\langle A_{i}\left(x^{*}\right), y-x^{*}\right\rangle \geq 0, \forall y \in C, \forall i=1, \ldots, N \\
\text { and } u^{*}=A x^{*} \in Q \text { solves } \\
\left\langle B_{j}\left(u^{*}\right), u-u^{*}\right\rangle \geq 0, \forall u \in Q, \forall j=1, \ldots, M,
\end{array}\right.
$$

where $C \subset H_{1}, Q \subset H_{2}$ are nonempty closed convex sets, $A_{i}: C \rightarrow H_{1}$, $B_{j}: Q \rightarrow H_{2}$ are nonlinear operators and $A: H_{1} \rightarrow H_{2}$ is a bounded linear operator. The solution set of Problem (4.1) is denoted by

$$
\Omega=\left\{x^{*} \in \cap_{i=1}^{N} V I\left(A_{i}, C\right): A x^{*} \in \cap_{j=1}^{M} V I\left(B_{j}, Q\right)\right\} .
$$

The authors in [7] used the gradient method to propose the parallel algorithm [7, Algorithm 6.4] for solving SVIP (4.1) and they proved that the sequences generated by the proposed algorithm converge weakly to some point in $\Omega$. However, in order to obtain this convergence, the method requires the restrictive condition that the operators $A_{i}, B_{j}$ are inverse strongly monotone. In this section, for solving Problem (4.1) we assume the operators $A_{i}, B_{j}$ satisfy the following condition.

\section{Condition 3}

- $A_{i}$ is pseudomonotone on $C$, i.e.,

$$
\left\langle A_{i}(x), y-x\right\rangle \geq 0 \Longrightarrow\left\langle A_{i}(y), x-y\right\rangle \leq 0, \forall x, y \in C
$$

- $\quad A_{i}$ is $L$-Lipschitz continuous on $C$, i.e., there exists a positive constant $L$ such that

$$
\left\|A_{i}(x)-A_{i}(y)\right\| \leq L\|x-y\|, \forall x, y \in C
$$


- $\quad B_{j}$ is monotone on $Q$, i.e.,

$$
\left\langle B_{j}(u)-B_{j}(v), u-v\right\rangle \geq 0, \forall u, v \in Q .
$$

Moreover, for obtaining the result of the weak convergence (Theorem 3 below), we need the following additional assumption

$$
A_{i}\left(x_{n}\right) \rightarrow A_{i}(x), i=1, \ldots, N
$$

for each sequence $\left\{x_{n}\right\} \subset C$ converging weakly to $x$. Hypothesis (4.2) is not necessary to establish the strong convergence (Theorem 4 below). We have the following lemma.

Lemma 6. Assume that the operators $A_{i}, B_{j}$ satisfy Condition 3. Then

i. The bifunction $f_{i}(x, y)=\left\langle A_{i}(x), y-x\right\rangle$ for all $x, y \in C$ satisfies Condition $1 a$ and if condition (4.2) holds then $f_{i}$ satisfies Condition 1. Besides, for each $\lambda \in\left(0, \frac{1}{L}\right), y^{i}=P_{C}\left(x-\lambda A_{i}(z)\right)$ iff

$$
y^{i}=\arg \min \left\{\lambda f_{i}(z, y)+\frac{1}{2}\|y-x\|^{2}: y \in C\right\} .
$$

ii. The bifunction $F_{j}(u, v)=\left\langle B_{j}(u), v-u\right\rangle$ for all $u, v \in Q$ satisfies Condition 2 and, for each $r>0, w^{j}=T_{r}^{F_{j}}(u)$ iff

$$
\left\langle w^{j}+r B_{j}\left(w^{j}\right)-u, v-w^{j}\right\rangle \geq 0, \forall v \in Q .
$$

Proof. $\quad$ i. The bifunction $f_{i}$ satisfies automatically the assumptions (A1), (A3a), (A4) in Condition 1a . It follows from the $L$ - Lipschitz continuity of $A_{i}$, the Cauchy-Schwarz and Cauchy inequalities that

$$
\begin{aligned}
f_{i}(x, y)+f_{i}(y, z)-f_{i}(x, z) & =\left\langle A_{i}(x)-A_{i}(y), y-z\right\rangle \geq-\left\|A_{i}(x)-A_{i}(y)\right\|\|y-z\| \\
& \geq-L\|x-y\|\|y-z\| \geq-\frac{L}{2}\|x-y\|^{2}-\frac{L}{2}\|y-z\|^{2} .
\end{aligned}
$$

Hence, $f_{i}$ is Lipschitz-type continuous on $C$ with $c_{1}=c_{2}=L / 2$ or (A2) holds for $f_{i}$. Thus, $f_{i}$ satisfies Condition 1a. Similarly, if condition (4.2) holds then $f_{i}$ satisfies Condition 1. By the definitions of $f_{i}$ and $y^{i}$,

$$
\begin{aligned}
y^{i} & =\underset{y \in C}{\operatorname{argmin}}\left\{\lambda\left\langle A_{i}(z), y-z\right\rangle+\frac{1}{2}\|y-x\|^{2}\right\} \\
& =\underset{y \in C}{\operatorname{argmin}}\left\{\frac{1}{2}\left\|y-\left(x-\lambda A_{i}(z)\right)\right\|^{2}-\frac{\lambda^{2}}{2}\left\|A_{i}(z)\right\|^{2}-\lambda\left\langle A_{i}(z), z-x\right\rangle\right\} \\
& =\underset{y \in C}{\operatorname{argmin}}\left\{\frac{1}{2}\left\|y-\left(x-\lambda A_{i}(z)\right)\right\|^{2}\right\}=P_{C}\left(x-\lambda A_{i}(z)\right),
\end{aligned}
$$

in which the third equality is followed from the fact that $\arg \min \{g(y)+a: y \in C\}=\arg \min \{g(y): y \in C\}$ for all $a \in \Re$. 
ii. By the hypothesis of $B_{j}$ and the definition of $F_{j}$, we see that $F_{j}$ satisfies immediately Condition 2. It follows from the definitions of $F_{j}, T_{r}^{F_{j}}$ and $w^{j}=$ $T_{r}^{F_{j}}(u)$ that

$$
\left\langle B_{j}\left(w^{j}\right), v-w^{j}\right\rangle+\frac{1}{r}\left\langle v-w^{j}, w^{j}-u\right\rangle \geq 0, \quad \forall v \in Q .
$$

This is equivalent to

$$
\left\langle w^{j}+r B_{j}\left(w^{j}\right)-u, v-w^{j}\right\rangle \geq 0, \forall v \in Q
$$

Lemma 6 is proved.

From Lemma 6 and Theorems 1, 2, we obtain the following results.

Theorem 3. Assume that $A_{i}, B_{j}$ are the operators satisfying Condition 3 and (4.2), $A: H_{1} \rightarrow H_{2}$ is a bounded linear operator with the adjoint $A^{*}$. In addition the solution set $\Omega$ of (4.1) is nonempty. Let $\left\{x_{n}\right\}$ be the sequence generated by the following manner: $x_{0} \in C$ and

$$
\left\{\begin{array}{l}
y_{n}^{i}=P_{C}\left(x_{n}-\lambda A_{i}\left(x_{n}\right)\right), \\
z_{n}^{i}=P_{C}\left(x_{n}-\lambda A_{i}\left(y_{n}^{i}\right)\right) \\
\left\langle w_{n}^{j}+r_{n} B_{j}\left(w_{n}^{j}\right)-\bar{z}_{n}, z-w_{n}^{j}\right\rangle \geq 0, \forall z \in Q \\
x_{n+1}=P_{C}\left(\bar{z}_{n}+\mu A^{*}\left(\bar{w}_{n}-A \bar{z}_{n}\right)\right),
\end{array}\right.
$$

where $\bar{z}_{n}$ and $\bar{w}_{n}$ are chosen as in Algorithm 1. Then, if $\lambda \in\left(0, \frac{1}{L}\right), r_{n} \geq d>0$, and $\mu \in\left(0, \frac{2}{\|A\|^{2}}\right)$ then $\left\{x_{n}\right\}$ converges weakly to some element in $\Omega$.

Theorem 4. Assume that $A_{i}, B_{j}$ are the operators satisfying Condition 3 and $A: H_{1} \rightarrow H_{2}$ is a bounded linear operator with the adjoint $A^{*}$. In addition the solution set $\Omega$ of (4.1) is nonempty. Let $\left\{x_{n}\right\}$ be the sequence generated by the following manner: $x_{0} \in C, C_{0}=C$, and

$$
\left\{\begin{array}{l}
y_{n}^{i}=P_{C}\left(x_{n}-\lambda A_{i}\left(x_{n}\right)\right) \\
z_{n}^{i}=P_{C}\left(x_{n}-\lambda A_{i}\left(y_{n}^{i}\right)\right) \\
\left\langle w_{n}^{j}+r_{n} B_{j}\left(w_{n}^{j}\right)-\bar{z}_{n}, z-w_{n}^{j}\right\rangle \geq 0, \forall z \in Q \\
t_{n}=P_{C}\left(\bar{z}_{n}+\mu A^{*}\left(\bar{w}_{n}-A \bar{z}_{n}\right)\right) \\
C_{n+1}=\left\{v \in C_{n}:\left\|t_{n}-v\right\| \leq\left\|\bar{z}_{n}-v\right\| \leq\left\|x_{n}-v\right\|\right\} \\
x_{n+1}=P_{C_{n+1}}\left(x_{0}\right)
\end{array}\right.
$$

where $\bar{z}_{n}, \bar{w}_{n}, \lambda, r_{n}$ and $\mu$ are defined as in Theorem 3. Then, the sequence $\left\{x_{n}\right\}$ converges strongly to $P_{\Omega}\left(x_{0}\right)$.

\section{A numerical example}

In this section, we consider $H_{1}=\Re^{m}, H_{2}=\Re,(m=1,5,10,100,500)$ and the feasible sets $C, Q$ are defined by

$$
C=\left\{x \in \Re^{m}: \sum_{i=1}^{m} x_{i} \geq-1, \quad-5 \leq x_{i} \leq 5, i=1, \ldots, m\right\}
$$


and $Q=[-1, \infty)$. The bifunctions $f_{i}: C \times C \rightarrow \Re, i=1, \ldots, N(N=$ $2,50,100)$ which come from the Nash-Cournot equilibrium model in [31]. They are defined by

$$
f_{i}(x, y)=\left\langle P_{i} x+Q_{i} y+q_{i}, y-x\right\rangle,
$$

where $q_{i} \in \Re^{m}, P_{i}, Q_{i} \in \Re^{m \times m}$ are two matrices of order $m$ such that $Q_{i}$ is symmetric, positive semidefinite and $Q_{i}-P_{i}$ is negative semidefinite. In this case, the bifunction $f_{i}$ satisfies both Condition 1 and Condition 1 a with the Lipschitz-type constants $c_{1}^{i}=c_{2}^{i}=\frac{1}{2}\left\|Q_{i}-P_{i}\right\|$, see [31, Lemma 6.2]. We chose $c_{1}=c_{2}=\max \left\{c_{1}^{i}: i=1, \ldots, N\right\}$ and $\lambda=\frac{1}{4 c_{1}}$.

The linear operator $A: \Re^{m} \rightarrow \Re$ is defined by $A x=\langle a, x\rangle$ where $a$ is a vector in $\Re^{m}$ whose elements are randomly generated in $[1, m]$. Thus, $A^{*} y=y . a$ for all $y \in \Re$ and $\|A\|=\|a\|$. We chose $\mu=\frac{1}{2\|a\|^{2}}$ and consider two bifunctions $F_{1}, F_{2}: Q \times Q \rightarrow \Re$ as $F_{1}(x, y)=x(y-x)$ and $F_{2}(x, y)=\left(2 x-x^{2}\right)(y-x)$ for all $x, y \in Q$. It is easy to show that $F_{1}, F_{2}$ satisfy Condition 2 and $\operatorname{EP}\left(F_{1}, Q\right)=$ $E P\left(F_{2}, Q\right)=\{0\}$. The starting point is chosen as $x_{0}=(1,1, \ldots, 1)^{T} \in \Re^{m}$. In Step 1 of Algorithms 1 and 2, we need to solve the following optimization program

$$
\arg \min \left\{\lambda f_{i}\left(x_{n}, y\right)+\frac{1}{2}\left\|x_{n}-y\right\|^{2}: y \in C\right\}
$$

or the following convex quadratic problem

$$
\arg \min \left\{\frac{1}{2} y^{T} H_{i} y+b_{i}^{T} y: y \in C\right\}
$$

where $H_{i}=2 \lambda Q_{i}+I$ and $b_{i}=\lambda\left(P_{i} x_{n}-Q_{i} x_{n}+q_{i}\right)-x_{n}$. Problem (5.2) can be effectively solved, for instance, by the MATLAB Optimization Toolbox to obtain the approximation $y_{n}^{i}$. Similarly, $z_{n}^{i}$ solves the following program

$$
\arg \min \left\{\frac{1}{2} y^{T} \widehat{H}_{i} y+\widehat{b}_{i}^{T} y: y \in C\right\}
$$

where $\widehat{H}_{i}=H_{i}$ and $\widehat{b}_{i}=\lambda\left(P_{i} y_{n}^{i}-Q_{i} y_{n}^{i}+q_{i}\right)-x_{n}$. Thus, the furthest element $\bar{z}_{n}$ from $x_{n}$ among all $z_{n}^{i}$ is chosen, and so $A \bar{z}_{n}=\left\langle a, \bar{z}_{n}\right\rangle$. In this example, we chose $r_{n}=1$ for all $n \geq 0$. From Step 3 of Algorithms 1 and 2, we have $w_{n}^{1}=T_{r_{n}}^{F_{1}}\left(A \bar{z}_{n}\right)$ or find $w_{n}^{1} \in Q$ such that

$$
\left(z-w_{n}^{1}\right)\left(2 w_{n}^{1}-A \bar{z}_{n}\right) \geq 0, \forall z \in Q .
$$

This is equivalent to $w_{n}^{1}=\frac{1}{2} A \bar{z}_{n}$. Similarly, we also obtain $w_{n}^{2}=\frac{3-\sqrt{9-4 A \bar{z}_{n}}}{2}$. From these relations, we can choose $\bar{w}_{n}$ which is the furthest element from $A \bar{z}_{n}$. Moreover,

$$
x_{n+1}=P_{C}\left(\bar{z}_{n}+\mu A^{*}\left(\bar{w}_{n}-A \bar{z}_{n}\right)\right)=P_{C}\left(\bar{z}_{n}+\mu\left(\bar{w}_{n}-A \bar{z}_{n}\right) a\right) .
$$

This means that $x_{n+1}$ solves the following distance optimization program

$$
\arg \min \left\{\left\|\bar{z}_{n}+\mu\left(\bar{w}_{n}-A \bar{z}_{n}\right) a-y\right\|^{2}: y \in C\right\}
$$


or $x_{n+1}$ solves the following problem

$$
\arg \min \left\{\frac{1}{2} y^{T} y+b^{T} y: y \in C\right\},
$$

where $b=-\bar{z}_{n}-\mu\left(\bar{w}_{n}-A \bar{z}_{n}\right) a$. Problem (5.3) is similarly solved to Problem (5.2). In numerical tests under, we chose $q_{i}$ being the zero vector, the elements of vector $a$ are randomly generated in $[1, m]$ and $P_{i}, Q_{i}$ are randomly generated matrixes as follows: We randomly chose $\lambda_{1 k}^{i} \in[-m, 0], \lambda_{2 k}^{i} \in[1, m], k=$ $1, \ldots, m, i=1 \ldots, N$. Set $\widehat{Q}_{1}^{i}, \widehat{Q}_{2}^{i}$ as two diagonal matrixes with eigenvalues $\left\{\lambda_{1 k}^{i}\right\}_{k=1}^{m}$ and $\left\{\lambda_{2 k}^{i}\right\}_{k=1}^{m}$, respectively. Then, we make a positive definite matrix $Q_{i}$ and a negative semidefinite matrix $T_{i}$ by using random orthogonal matrixes with $\widehat{Q}_{2}^{i}$ and $\widehat{Q}_{1}^{i}$, respectively. Finally, set $P_{i}=Q_{i}-T_{i}$. It is easy to see that $\cap_{i=1}^{N} E P\left(f_{i}, C\right)$ consists of the zero vector $0 \in \Re^{m}$. Therefore, $\Omega=\{0\}$. The stopping criteria is defined as $\left\|x_{n}-x^{\dagger}\right\|=\left\|x_{n}\right\| \leq$ TOL. The numerical results for Algorithm 1 are showed in Table 1 for the time of execution in second $(\mathrm{CPU}(\mathrm{s}))$ and the number of iterations (Iter.). The experiments are performed on a PC Desktop Intel(R) Core(TM) i5-3210M CPU @ 2.50GHz $2.50 \mathrm{GHz}$, RAM 2.00 GB.

Table 1. Experiment for Algorithm 1

\begin{tabular}{|c|c|c|c|c|c|c|c|}
\hline \multirow[b]{2}{*}{$\mathrm{m}$} & \multirow[b]{2}{*}{ TOL } & \multicolumn{2}{|c|}{$\mathrm{N}=2$} & \multicolumn{2}{|c|}{$\mathrm{N}=50$} & \multicolumn{2}{|c|}{$\mathrm{N}=100$} \\
\hline & & CPU(s) & Iter. & CPU(s) & Iter. & $\mathrm{CPU}(\mathrm{s})$ & Iter. \\
\hline \multirow{2}{*}{1} & $10^{-3}$ & 0.061 & 6 & 3.754 & 6 & 10.611 & 6 \\
\hline & $10^{-6}$ & 0.222 & 10 & 5.565 & 10 & 23.345 & 10 \\
\hline \multirow{2}{*}{5} & $10^{-3}$ & 0.158 & 4 & 2.373 & 4 & 9.578 & 4 \\
\hline & $10^{-6}$ & 0.244 & 7 & 3.574 & 6 & 15.659 & 6 \\
\hline \multirow{2}{*}{10} & $10^{-3}$ & 0.176 & 4 & 2.494 & 4 & 6.614 & 4 \\
\hline & $10^{-6}$ & 0.228 & 7 & 3.289 & 6 & 14.118 & 6 \\
\hline \multirow{2}{*}{100} & $10^{-3}$ & 0.273 & 4 & 5.384 & 4 & 14.135 & 4 \\
\hline & $10^{-6}$ & 0.405 & 7 & 8.758 & 6 & 22.281 & 6 \\
\hline \multirow{2}{*}{500} & $10^{-3}$ & 2.605 & 5 & 52.877 & 5 & 113.215 & 6 \\
\hline & $10^{-6}$ & 4.041 & 8 & 72.749 & 7 & 149.628 & 7 \\
\hline
\end{tabular}

It has been proved that Algorithm 2 is strongly convergent. Theoretically, it is useful in infinite dimensional Hilbert spaces because in practice, it is not easy to construct the sets $\left\{C_{n}\right\}$. However, we would like to illustrate its convergence in this numerical example and have not the intent for comparing Algorithm 1 with Algorithm 2. It is clear that the set $C$ defined by (5.1) as a polyhedral convex set and it is formulated by $C=\left\{x \in \Re^{m}: A_{0} x \leq b_{0}\right\}$, where $b_{0}=$ $(1,5,5, \ldots, 5)^{T} \in \Re^{m}$ and $A_{0}=\left\{a_{i j}\right\} \in \Re^{(2 m+1) \times m}$ with $a_{1 j}=-1$ for all $j=1, \ldots, m$ and

$$
a_{i j}= \begin{cases}1 & \text { if } i=2 j \\ -1 & \text { if } i=2 j+1 \\ 0 & \text { otherwise }\end{cases}
$$


We have $C_{0}=C=\left\{x \in \Re^{m}: A_{0} x \leq b_{0}\right\}$ and $C_{n+1}=C_{n} \cap C_{n}^{1} \cap C_{n}^{2}$, where

$$
\begin{aligned}
C_{n}^{1} & =\left\{x \in \Re^{m}:\left\|t_{n}-x\right\| \leq\left\|\bar{z}_{n}-x\right\|\right\} \\
& =\left\{x \in \Re^{m}: 2\left\langle\bar{z}_{n}-t_{n}, x\right\rangle \leq\left\|\bar{z}_{n}\right\|^{2}-\left\|t_{n}\right\|^{2}\right\}, \\
C_{n}^{2} & =\left\{x \in \Re^{m}:\left\|\bar{z}_{n}-x\right\| \leq\left\|x_{n}-x\right\|\right\} \\
& =\left\{x \in \Re^{m}: 2\left\langle x_{n}-\bar{z}_{n}, x\right\rangle \leq\left\|x_{n}\right\|^{2}-\left\|\bar{z}_{n}\right\|^{2}\right\} .
\end{aligned}
$$

We denote $\bar{A}_{n}$ by the matrix of the size $2 \times m$ and $\bar{a}_{n}$ by the vector as

$$
\bar{A}_{n}=\left(\begin{array}{c}
2\left(\bar{z}_{n}-t_{n}\right)^{T} \\
2\left(x_{n}-\bar{z}_{n}\right)^{T}
\end{array}\right), \bar{a}_{n}=\left(\left\|\bar{z}_{n}\right\|^{2}-\left\|t_{n}\right\|^{2},\left\|x_{n}\right\|^{2}-\left\|\bar{z}_{n}\right\|^{2}\right)^{T} .
$$

Thus, $C_{n}^{1} \cap C_{n}^{2}=\left\{x \in \Re^{m}: \bar{A}_{n} x \leq \bar{a}_{n}\right\}$.

Assume that $C_{n}=\left\{x \in \Re^{m}: A_{n} x \leq b_{n}\right\}$, by setting

$$
A_{n+1}=\left(\begin{array}{c}
A_{n} \\
\bar{A}_{n}
\end{array}\right), b_{n+1}=\left(\begin{array}{c}
b_{n} \\
\bar{a}_{n}
\end{array}\right) \text {. }
$$

Then $C_{n+1}=\left\{x \in \Re^{m}: A_{n+1} x \leq b_{n+1}\right\}$. From Step 6 of Algorithm 2, $x_{n+1}=$ $P_{C_{n+1}}\left(x_{0}\right)$, i.e., $x_{n+1}$ solves the following optimization problem

$$
\arg \min \left\{\frac{1}{2} y^{T} y+x_{0}^{T} y: y \in C_{n+1}\right\} .
$$

Problem (5.4) is effectively solved by the MATLAB optimization Toolbox. Note

\begin{tabular}{|c|c|c|c|c|c|c|c|}
\hline \multirow[b]{2}{*}{$\mathrm{m}$} & \multirow[b]{2}{*}{ TOL } & \multicolumn{2}{|c|}{$\mathrm{N}=2$} & \multicolumn{2}{|c|}{$\mathrm{N}=50$} & \multicolumn{2}{|c|}{$\mathrm{N}=100$} \\
\hline & & $\mathrm{CPU}(\mathrm{s})$ & Iter. & $\mathrm{CPU}(\mathrm{s})$ & Iter. & $\mathrm{CPU}(\mathrm{s})$ & Iter. \\
\hline \multirow{2}{*}{1} & $10^{-3}$ & 0.102 & 6 & 2.027 & 6 & 5.783 & 6 \\
\hline & $10^{-6}$ & 0.351 & 11 & 3.905 & 11 & 8.329 & 11 \\
\hline \multirow{2}{*}{5} & $10^{-3}$ & 1.394 & 54 & 16.468 & 41 & 33.844 & 29 \\
\hline & $10^{-6}$ & 3.909 & 127 & 49.388 & 114 & 99.714 & 113 \\
\hline \multirow{2}{*}{10} & $10^{-3}$ & 5.216 & 139 & 65.798 & 128 & 127.887 & 122 \\
\hline & $10^{-6}$ & 15.42 & 402 & 209.645 & 370 & 347.256 & 363 \\
\hline \multirow{2}{*}{100} & $10^{-3}$ & Slow conv. & - & - & - & - & - \\
\hline & $10^{-6}$ & Slow conv. & - & - & - & - & - \\
\hline
\end{tabular}
that the number of the constrained linear inequalities in $C_{n+1}$ increases by the number of iterations $n$, in fact, the matrix $A_{n+1}$ has the size $(2 m+2 n+3) \times m$. This might affect the efficiency of solving Problem (5.4) when the numbers $m, n$ are large. The numerical experiments for Algorithm 2 are presented in Table 2. In this case, we see that Algorithm 2 converges very slowly with $m=100$.

Table 2. Experiment for Algorithm 2

We also perform the numerical experiments for Algorithms 1 and 2 with a same data generated randomly. The results are showed in Table 3. 
Table 3. Experiment for Algorithms 1 and 2 with a same data

\begin{tabular}{|c|c|c|c|c|c|c|c|c|c|}
\hline \multirow[b]{3}{*}{$\mathrm{m}$} & \multirow[b]{3}{*}{ TOL } & \multicolumn{4}{|c|}{$\mathrm{N}=50$} & \multicolumn{4}{|c|}{$\mathrm{N}=100$} \\
\hline & & \multicolumn{2}{|c|}{ Alg. 1} & \multicolumn{2}{|c|}{ Alg. 2} & \multicolumn{2}{|c|}{ Alg. 1} & \multicolumn{2}{|c|}{ Alg. 2} \\
\hline & & $\mathrm{CPU}(\mathrm{s})$ & Iter. & $\mathrm{CPU}(\mathrm{s})$ & Iter. & $\mathrm{CPU}(\mathrm{s})$ & Iter. & $\mathrm{CPU}(\mathrm{s})$ & Iter. \\
\hline \multirow{2}{*}{1} & $10^{-3}$ & 2.056 & 6 & 2.272 & 6 & 8.712 & 6 & 6.691 & 6 \\
\hline & $10^{-6}$ & 3.253 & 10 & 4.109 & 11 & 14.462 & 10 & 16.473 & 11 \\
\hline \multirow[b]{2}{*}{5} & $10^{-3}$ & 1.317 & 4 & 22.881 & 52 & 5.044 & 4 & 60.364 & 39 \\
\hline & $10^{-6}$ & 2.974 & 7 & 60.375 & 126 & 8.008 & 6 & 180.072 & 119 \\
\hline \multirow{2}{*}{10} & $10^{-3}$ & 1.517 & 4 & 59.724 & 112 & 4.945 & 4 & 209.523 & 142 \\
\hline & $10^{-6}$ & 2.644 & 6 & 210.139 & 383 & 8.787 & 6 & 622.079 & 417 \\
\hline
\end{tabular}

\section{Conclusions}

We have proposed two parallel extragradient-proximal algorithms for split equilibrium problems and proved their convergence. We have designed the algorithms by combining the extragradient method for a class of pseudomonotone and Lipschitz-type continuous bifunctions, the proximal method for monotone bifunctions and the shrinking projection method. The numerical experiments are implemented for bifunctions which are generalized from the Nash-Cournot equilibrium model to illustrate the convergence of the proposed algorithms.

\section{Acknowledgements}

The author would like to thank the Associate Editor and the anonymous referees for their valuable comments and suggestions which helped us very much in improving the original version of this paper.

\section{References}

[1] P.N. Anh. A hybrid extragradient method extended to fixed point problems and equilibrium problems. Optimization, 62(2):271-283, 2013. http://dx.doi.org/10.1080/02331934.2011.607497.

[2] H. Attouch, A. Cabot A, F. Frankel and J. Peypouquet. Alternating proximal algorithms for constrained variational inequalities. application to domain decomposition for PDE's. Nonlinear Anal. TMA, 74(18):7455-7473, 2011. http://dx.doi.org/10.1016/j.na.2011.07.066.

[3] E. Blum and W. Oettli. From optimization and variational inequalities to equilibrium problems. Math. Program., 63:123-145, 1994.

[4] C. Byrne. A unified treatment of some iterative algorithms in signal processing and image reconstruction. Inverse Probl., 20(1):103-120, 2004. http://dx.doi.org/10.1088/0266-5611/20/1/006.

[5] Y. Censor, M. D. Altschule and W. D. Powlis. On the use of Cimmino's simultaneous projections method for computing a solution of the inverse problem in radiation therapy treatment planning. Inverse Probl., 4(3):607-623, 1988. http://dx.doi.org/10.1088/0266-5611/4/3/006. 
[6] Y. Censor, T. Bortfeld and A. Trofimov. B. Martin. A unified approach for inversion problems in intensity-modulated radiation therapy. Phys. Med. Biol., 51(10):2353-2365, 2006. http://dx.doi.org/10.1088/0031-9155/51/10/001.

[7] Y. Censor, A. Gibali and S. Reich. Algorithms for the split variational inequality problem. Numer. Algorithms, 59(0):301-323, 2012. http://dx.doi.org/10.1007/s11075-011-9490-5.

[8] Y. Censor and A. Segal. The split common fixed point problemfor directed operators. J. Convex Anal., 16:587-600, 2009.

[9] S. Chang, L. Wang, X.R. Wang and G. Wang. General split equality equilibrium problems with application to split optimization problems. J. Optim. Theory Appl., 166(2):377-390, 2015. http://dx.doi.org/10.1007/s10957-015-0739-3.

[10] P.L. Combettes and S.A. Hirstoaga. Equilibrium programming in Hilbert spaces. J. Nonlinear Convex Anal., 6(1):117-136, 2005.

[11] J. Deepho and P. Kumam. The modified Mann's type extragradient for solving split feasibility and fixed point problems of Lipschitz asymptotically quasi nonexpansive mappings. Fixed Point Theory Appl., 2013:349, 2013.

[12] J. Deepho and P. Kumam. The hybrid steepest descent method for split variational inclusion and constrain convex minimization problems. Abstract and Applied Analysis, Article ID: 365203 (13 pages), 2014, 2014.

[13] J. Deepho, W. Kumm and P. Kumm. A new hybrid projection algorithm for solving the split generalized equilibrium problems and the system of variational inequality problems. J. Math. Model. Algor., 13(4):405-423, 2014. http://dx.doi.org/10.1007/s10852-014-9261-0.

[14] J. Deepho, J. Martnez-Moreno, K. Sitthithakerngkiet and P. Kumam. Convergence analysis of hybrid projection with Cesaro mean method for the split equilibrium and general system of finite variational inequalities. J. Comput. Appl. Math, 2015. http://dx.doi.org/10.1016/j.cam.2015.10.006.

[15] F. Facchinei and J. S. Pang. Finite-Dimensional Variational Inequalities and Complementarity Problems. Springer, Berlin, 2003.

[16] K. Goebel and S. Reich. Uniform convexity, hyperbolic geometry, and nonexpansive mappings. Marcel Dekker, New York, 1984.

[17] Z. He. The split equilibrium problems and its convergence algorithms. J. Inequal. Appl., 2012, 2012. http://dx.doi.org/10.1186/1029-242x-2012-162.

[18] D.V. Hieu, L.D. Muu and P.K. Anh. Parallel hybrid extragradient methods for pseudomonotone equilibrium problems and nonexpansive mappings. Numer. Algor., pp. 1-21, 2016. http://dx.doi.org/10.1007/s11075-015-0092-5.

[19] K.R. Kazmi and S.H. Rizvi. Iterative approximation of a common solution of a split equilibrium problem, a variational inequality problem and a fixed point problem. J. Egyptian Math. Society, 21(1):44-51, 2013. http://dx.doi.org/10.1016/j.joems.2012.10.009.

[20] K.R. Kazmi and S.H. Rizvi. Implicit iterative method forapproximating a common solution of split equilibrium problem and fixed point problem for a nonexpansive semigroup. Arab J. Math. Sci., 20(1):57-75, 2014. http://dx.doi.org/10.1016/j.ajmsc.2013.04.002.

[21] I.V. Konnov. Combined Relaxation Methods for Variational Inequalities. Springer, Berlin, 2000. 
[22] W. Kumam, J. Deepho and P. Kumam. Hybrid extragradient method for finding a common solution of the split feasibility and a system of equilibrium problems. Dynamics of Continuous, Discrete and Impulsive System, DCDIS Series B: Applications \& Algorithms, 21(6):367-388, 2014.

[23] G. Mastroeni. On auxiliary principle for equilibrium problems. In: Daniele P, Giannessi F, Maugeri A, editors. E quilibrium problems and variational models, volume 68. Dordrecht: Kluwer Academic, 2003. http://dx.doi.org/10.1007/9781-4613-0239-1_15.

[24] A. Moudafi. Split monotone variational inclusions. J Optim Theory App, 150(2):275-283, 2011. http://dx.doi.org/10.1007/s10957-011-9814-6.

[25] A. Moudafi. Eman Al-Shemas: simultaneously iterative methods for split equality problem. Trans. Math. Program. Appl., 1(0):1-11, 2013.

[26] A. Moudafi. A relaxed alternating CQ algorithm for convex feasibility problems. Nonlinear Anal. TMA, 79:117-121, 2013. http://dx.doi.org/10.1016/j.na.2012.11.013.

[27] L.D. Muu and W. Oettli. Convergence of an adative penalty scheme for finding constrained equilibria. Nonlinear Anal. TMA, 18(12):1159-1166, 1992. http://dx.doi.org/10.1016/0362-546X(92)90159-C.

[28] T.T.V. Nguyen, J.J. Strodiot and V.H. Nguyen. Hybrid methods for solving simultaneously an equilibrium problem and countably many fixed point problems in a Hilbert space. J. Optim. Theory Appl., 160(3):809-831, 2014. http://dx.doi.org/10.1007/s10957-013-0400-y.

[29] Z. Opial. Weak convergence of the sequence of successive approximations for nonexpansive mappings. Bull. Amer. Math. Soc., 73:591-597, 1967. http://dx.doi.org/10.1090/S0002-9904-1967-11761-0.

[30] X. Qin, Y. J. Cho and S. M. Kang. Convergence analysis on hybrid projection algorithms for equilibrium problems and variational inequality problems. Model. Math. Anal., 14(3):335-351, 2009. http://dx.doi.org/10.3846/13926292.2009.14.335-351.

[31] T.D. Quoc, L.D. Muu and N.V. Hien. Extragradient algorithms extended to equilibrium problems. Optimization, $\mathbf{5 7}(6): 749-776,2008$. http://dx.doi.org/10.1080/02331930601122876.

[32] K. Sitthithakerngkiet, J. Deepho and P. Kumam. A hybrid viscosity algorithm via modify the hybrid steepest descent method for solving the split variational inclusion and fixed point problems. Appl. Math. Comput., 250:986-1001, 2015. http://dx.doi.org/10.1016/j.amc.2014.10.130.

[33] P.T. Vuong, J.J. Strodiot and V.H. Nguyen. On extragradient-viscosity methods for solving equilibrium and fixed point problems in a Hilbert space. Optimization, 64(2):429-451, 2015. http://dx.doi.org/10.1080/02331934.2012.759327. 